\title{
Hydro and Jets in Relativistic Heavy-Ion Collisions
}

\author{
L.V. Bravina ${ }^{1,3,4, a}$, B.H. Brusheim Johansson ${ }^{1}$, J. Crkovská5, ${ }^{5,6}$, G.Kh. Eyyubova ${ }^{2,7}$, V.L. Korotkikh ${ }^{2}$, \\ I.P. Lokhtin ${ }^{2}$, L.V. Malinina ${ }^{2,7}$, E.N. Nazarova ${ }^{2}$, S.V. Petrushanko ${ }^{2}$, A.M. Snigirev², and \\ E.E. Zabrodin ${ }^{1,2,3,4}$
}

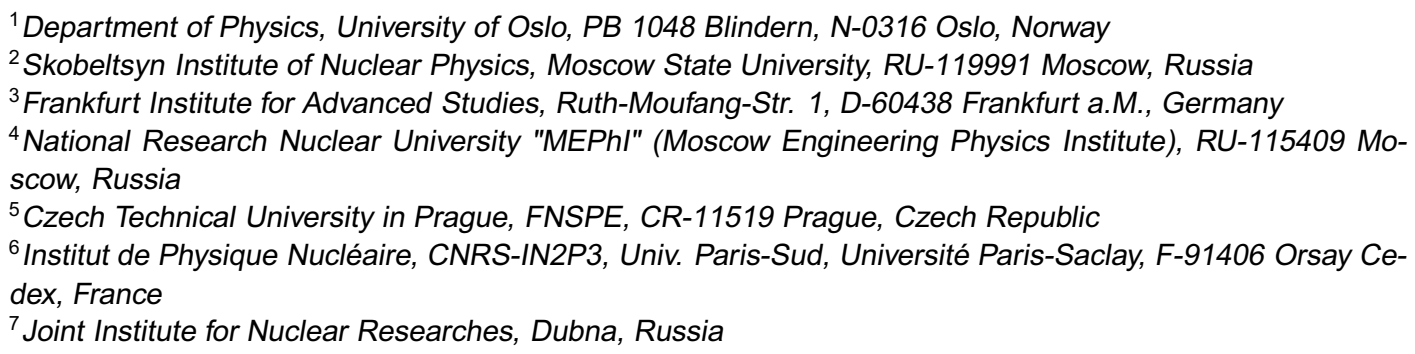

\begin{abstract}
We apply HYDJET++ model, which contains the treatment of both soft and hard processes, to study the heavy-ion collisions at LHC energies. The interplay of parametrised hydrodynamics and jets describes many features of the development of particle anisotropic flow including the break-up of mass hierarchy of elliptic and triangular flow, the falloff of the flow at certain transverse momentum and violation of the number-ofconstituent-quark (NCQ) scaling at LHC energies compared to the lower ones. Other signals, such as long-range dihadron correlations (ridge) and event-by-event (EbyE) fluctuations of the flow are also discussed. Model calculations demonstrate a good agreement with the available experimental data.
\end{abstract}

\section{Introduction}

The main difficulties emerging in analysis of data on relativistic heavy-ion collisions (HIC's) are (i) absence of the unified theory for the description of these collisions, (ii) that perturbative quantum chromodynamics (QCD) does not apply for "soft" interactions with small transverse momentum transfer, which play an essential role in physics of HIC's, and (iii) that the final state of HIC's is very complicated to extract easily the signatures of the new state of matter, the quark-gluon plasma (QGP). Therefore, models are indispensable for evaluating the current experiments and for planing the new ones. These models can be subdivided into macroscopic, i.e. thermal [1-3] and hydrodynamic models [4-6], and microscopic Monte Carlo models [7-10], incorporating partonic and hadronic degrees of freedom in a consistent fashion. Note that for better description of data, hydrodynamic models nowadays are employing the microscopic transport models as afterburner.

\footnotetext{
a e-mail: larissa.bravina@fys.uio.no
} 
In contrast to microscopic transport models, macroscopic models usually deal with soft processes with particle transverse momentum up to $p_{T} \approx 1.5-2 \mathrm{GeV} / c$ only, and do not include jets. HYDJET ++ model [11] was, probably, the first attempt to combine a hydro-like state and hard processes within a single event generator. This idea appears to be very promising. After the discussion of basic principles of the model in Sec. 2, the most interesting results obtained by the HYDJET++ are listed in Sec. 3. Finally, conclusions are drawn in Sec. 4.

\section{HYDrodynamics with JETs (HYDJET++)}

In HYDJET++ the soft state is represented by a hydro-like system $[12,13]$. It is coupled to a hard multiparton state, where the hard partons are suffering the collisional and gluon radiation losses [14] when propagating the dense and hot medium. For the sake of simplicity, both states are treated independently. In the soft sector sector HYDJET++ employs the popular Bjorken or Hubble expansion scenarios for the overlapping zone of heavy-ion collisions. At certain temperature $T=T^{c h}$ and chemical potentials $\mu_{i}$, where $i=B, S, Q$ represents baryon charge, strangeness and electric charge, respectively, the thermalised fireball breaks into individual hadrons in a sudden chemical freeze-out process. The particle number density $\rho_{i}^{e q}\left(T, \mu_{i}\right)$ is given by the well-known series expansion [4]

$$
\rho_{i}^{e q}\left(T, \mu_{i}\right)=\frac{g_{i}}{2 \pi^{2}} m_{i}^{2} T \sum_{k=1}^{\infty} \frac{(\mp)^{k+1}}{k} \exp \left(\frac{k \mu_{i}}{T}\right) K_{2}\left(\frac{k m_{i}}{T}\right),
$$

where $g_{i}$ is the spin degeneracy factor, $m_{i}$ is the particle mass, and $K_{2}$ is the modified Bessel function of a second kind. To calculate the particle yields one has to know the fireball volume. It is proportional to the mean number of wounded nucleons at a given impact parameter $b$, see [11-13] for details. After the chemical freeze-out, the system of hadrons continues to expand until the thermal contact between the particles is lost. This is the stage of thermal freeze-out. The only final-state interactions taken into account in the model are two- and three-body decays of resonances. HYDJET++ benefits from the extensive table of particles which contains more than 360 meson and baryon states including the charmed ones. The mass distribution of the resonances is given by a non-relativistic Breit-Wigner formula

$$
P(m) d m \propto \frac{1}{\left(m-m_{0}\right)^{2}+\Delta m^{2} / 4} d m,
$$

where $m_{0}$ and $\Delta m$ are the resonance nominal mass and width, respectively.

The hard multiparton stage emerges in the model as a superposition of individual hard nucleonnucleon (NN) collisions. For each collision, the PYQUEN routine [14] generates the initial parton spectra (by means of the tune Pro-Q20 of PYTHIA [15]) and the production vertexes. The parton rescattering stage is accompanied by collisional energy loss and radiation of gluons. In high-momentum transfer limit, the collisional loss reads

$$
\frac{d E^{c o l}}{d l}=\frac{1}{4 T \lambda \sigma} \int_{\mu_{D}^{2}}^{t_{\max }} d t \frac{d \sigma}{d t} t,
$$

with the dominant contribution to the differential scattering cross section

$$
\frac{d \sigma}{d t} \cong C \frac{2 \pi \alpha_{s}^{2}(t)}{t^{2}} \frac{E^{2}}{E^{2}-m_{p}^{2}} .
$$


Here $\lambda=1 /(\sigma \rho)$ is the mean free path of a parton in a medium with density $\rho$ and integral cross section of parton-medium interaction $\sigma$. In the last equation $E$ and $m_{p}$ are energy and mass of the parton, $\alpha_{s}$ is the QCD running coupling constant, and $C=4 / 9,1,9 / 4$ for $q q, g q$ and $g g$ interaction, respectively. Then, the energy spectrum of coherent medium-induced gluon radiation and the corresponding dominant part of radiative energy loss of massless parton in the framework of BDMS formalism $[16,17]$ become

$$
\begin{aligned}
& {\frac{d E^{r a d}}{d l}}^{r}=\frac{2 \alpha_{s}\left(\mu_{D}^{2}\right) C_{R}}{\pi L} \int_{\omega_{\min }}^{E} d \omega\left[1-y+\frac{y^{2}}{2}\right] \ln \left|\cos \left(\omega_{1} \tau_{1}\right)\right|, \\
& \omega_{1}=\sqrt{i\left(1-y+\frac{C_{R}}{3} y^{2}\right) \bar{\kappa} \ln \frac{16}{\bar{\kappa}}} \quad \text { with } \quad \bar{\kappa}=\frac{\mu_{D}^{2} \lambda_{g}}{\omega(1-y)},
\end{aligned}
$$

containing $\tau_{1}=L /\left(2 \lambda_{g}\right), \lambda_{g}$ is the gluon mean free path, and $y=\omega / E$ is the parton energy fraction carried away by the gluon. Partons produced in initial hard scatterings with the transverse momentum transfer lower than a certain limit $p_{\mathrm{T}}^{\min }$ are excluded from the hard component. Their hadronization products are added to the thermalised component of particle spectrum.

Let us discuss generation of the anisotropic flow in non-central heavy ion collisions in the model. Recall, that the flow harmonics are the Fourier coefficients of series expansion of the invariant cross section in azimuthal plane [18]

$$
\begin{aligned}
E \frac{d^{3} N}{d^{3} p} & =\frac{1}{\pi} \frac{d^{2} N}{d p_{T}^{2} d y}\left\{1+2 \sum_{n=1}^{\infty} v_{n} \cos \left[n\left(\phi-\Psi_{n}\right)\right]\right\}, \\
v_{n} & =\left\langle\cos \left[\left(n\left(\phi-\Psi_{n}\right)\right]\right\rangle .\right.
\end{aligned}
$$

Here $y$ is the particle rapidity, $\phi$ is the azimuthal angle between the particle transverse momentum $p_{T}$ and the participant plane, and $\Psi_{n}$ is the azimuth of the participant plane of $n$-th order. The flow harmonic coefficients are called directed, $v_{1}$, elliptic, $v_{2}$, triangular, $v_{3}$, quadrangular, $v_{4}$, pentagonal, $v_{5}$, hexagonal, $v_{6}$, flow and so forth. The transverse radius of the fireball for non-central heavy ion collision depends on impact parameter $b$, azimuthal angle $\phi$ and spatial eccentricity $\epsilon(b)=\left(R_{y}^{2}-\right.$ $\left.R_{x}^{2}\right) /\left(R_{y}^{2}+R_{x}^{2}\right)$ as [13]

$$
R_{\text {ell }}(b, \phi)=R_{\text {fr. }- \text { out }}(b) \sqrt{\frac{1-\epsilon^{2}(b)}{1+\epsilon(b) \cos 2 \phi}} .
$$

The parameter $R_{f r-\text { out }}(b)$ is linked to the freeze-out radius of the fireball in a central collision, $R_{0}$, as $R_{f r \text {-out }}(b)=R_{0} \sqrt{1-\epsilon(b)}$. The momentum anisotropy arises from the pressure gradients, which are stronger in the direction of short axis of the ellipsoid. Another free parameter, $\delta(b)$, connects the flow azimuthal angle $\phi_{f l}$ with the azimuthal angle $\phi$. Namely,

$$
\delta(b)=\frac{1-C^{2}}{1+C^{2}}, \quad C=\frac{\tan \phi_{f l}}{\tan \phi} .
$$

Triangular flow needs further modification of the transverse radius [20], which is controlled by new free parameter $\epsilon_{3}(b)$

$$
R_{\text {triang }}(b, \phi)=R_{\text {ell }}(b, \phi)\left\{1+\epsilon_{3}(b) \cos \left[3\left(\phi-\Psi_{3}\right)\right]\right\} .
$$

According to experimental data, elliptic and triangular flow are not correlated in the model. Further details of HYDJET++ can be found in [11-13]. 


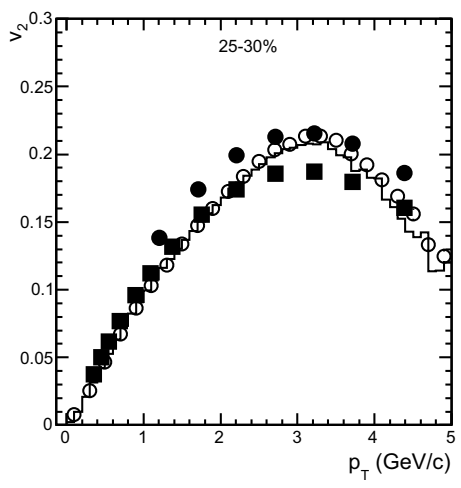

Figure 1. The $v_{2}\left(p_{T}\right)$ distributions in the HYDJET++ Figure 2. The $v_{3}\left(p_{T}\right)$ distributions for charged hadrons model for $25-30 \%$ centrality collisions (histogram) from (i) all processes (solid circles), (ii) soft+hard procompared to event-plane flow restoration method (open cesses w/o jet quenching (open triangles), (iii) directly circles) and CMS data (full symbols). See text for de- frozen hadrons (dash-dotted line), (iv) soft processes tails.

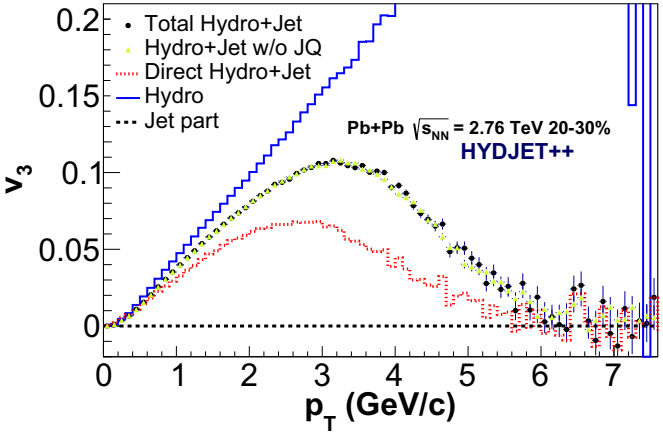

(solid histogram), (v) jets (dashed line) in $\mathrm{Pb}+\mathrm{Pb}$ at $2.76 \mathrm{TeV}$ with centrality $20-30 \%$.

\section{Interplay of soft processes and jets}

For our further analysis $\mathrm{Pb}+\mathrm{Pb}$ collisions at $\sqrt{s}=2.76 \mathrm{TeV}$ are chosen. Figure 1 displays elliptic flow of charged hadrons produced in collisions with centrality $25-30 \%$. Experimental data by the CMS Collaboration [19] obtained by the 2-cumulant and Lee-Yang zeroes (LYZ) methods are plotted also. To estimate possible distortion of the flow spectra caused by the reconstruction procedure, the generated particle spectra were used as an input for the flow determination. The event-plane (EP) method was employed. The restored spectrum is very close to the original one. Model calculations agree well with the data. The good agreement with the experiment is obtained for the triangular flow and higher flow harmonics as well, see [20].

Note that falloff of the differential flow $v_{2}\left(p_{T}\right)$ at $p_{T} \geq 3 \mathrm{GeV} / c$ is a common feature of all flow harmonics. Since we apply ideal parameterised hydrodynamics which has no such peculiarity, the most plausible explanation of the drop of $v_{n}\left(p_{T}\right)$ is the jet influence. One can see this in Fig. 2, which shows the partial contributions of different subprocesses to the triangular flow $v_{3}\left(p_{T}\right)$ in collisions with centrality $20-30 \%$. In addition to the total $v_{3}$, this figure presents also the flow in case of absence of jet-medium interaction, flow of particles produced merely in soft processes, flow of particles without the resonance feed-down, and flow of hadrons decoupled from jets. The latter is essentially zero, whereas its hydrodynamic counterpart increases with rising $p_{T}$. After certain transverse momentum, however, the total spectrum of hadrons is dominated by particles produced in hard processes which can carry very weak flow because of the jet quenching [21]. Since these particles prevail in the hadron spectrum, the total flow drops.

The same mechanism explains also violation of the meson-baryon hierarchy in $p_{T}$-differential $v_{n}\left(p_{T}\right)$, seen in Fig. 3. This figure depicts the triangular flow of most abundant charged hadrons, i.e. pions, $\pi^{ \pm}$, kaons, $K^{ \pm}$, and (anti)protons, $p+\bar{p}$. At transverse momenta below $2 \mathrm{GeV} / c$, triangular flow of mesons is stronger than the flow of $p+\bar{p}$. Moreover, in ideal hydrodynamics $v_{3}\left(p_{T}\right)$ of different hadron species do not cross each other. But then jets come into play. For a given hadron species the crossing point of hard and soft parts of its $p_{T}$-spectrum depends on the hadron mass. The heavier the hadron, the larger the transverse momentum at which the jet-produced particles become more 


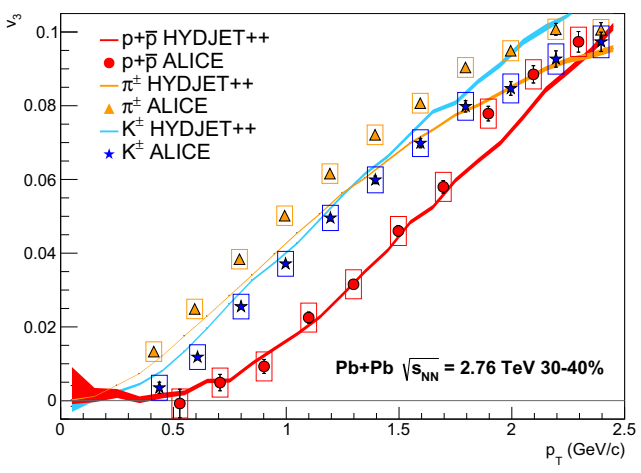

Figure 3. $p_{T}$-differential triangular flow of charged pions, kaons, and protons plus antiprotons in $\mathrm{Pb}+\mathrm{Pb}$ collisions at $2.76 \mathrm{TeV}$ with centrality $20-30 \%$. solid curves indicate HYDJET++ calculations, symbols show ALICE data from [22].

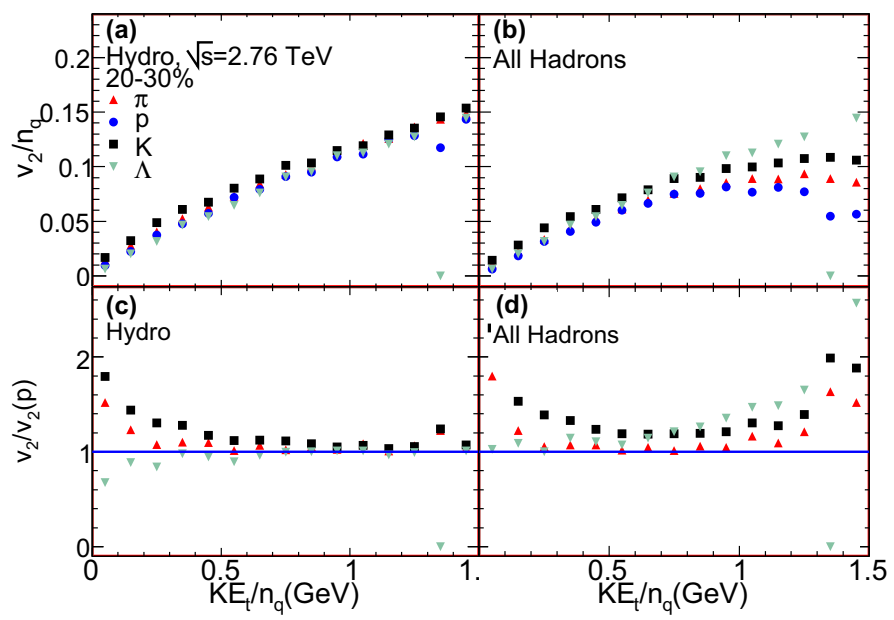

Figure 4. Upper row: The $K E_{\mathrm{T}} / n_{q}$ dependence of elliptic flow for (a) hadrons produced in soft processes only, and (b) all hadrons calculated in HYDJET ++ model for $\mathrm{Pb}+\mathrm{Pb}$ collisions at $2.76 \mathrm{TeV}$ with centrality $20-30 \%$. Bottom row: The distributions from the upper row are normalised to the elliptic flow of $p+\bar{p}$.

abundant. Therefore, the $p_{T}$-differential flow of mesons experiences the saturation and subsequent drop at smaller $p_{T}$ compared to heavier baryons. This effect is called violation of the mass ordering of the flow.

Also, increasing number of hard processes with the rise of collision energy of heavy ions explains the worsening of the number-of-constituent-quark scaling of elliptic flow at LHC. The effect was first observed in $\mathrm{Au}+\mathrm{Au}$ collisions at RHIC $[23,24]$. If the elliptic flow $v_{2}$ and the transverse kinetic energy $K E_{\mathrm{T}}=m_{\mathrm{T}}-m_{0}$ are divided by a number of constituent quarks $n_{q}, 3$ for baryons and 2 for mesons, then the distributions $v_{2} / n_{q}\left(K E_{\mathrm{T}} / n_{q}\right)$ for all hadrons coincide with good accuracy up to $K E_{\mathrm{T}} / n_{q} \approx 1 \mathrm{GeV}$. This circumstance was taken as a strong evidence of the predominant production of 


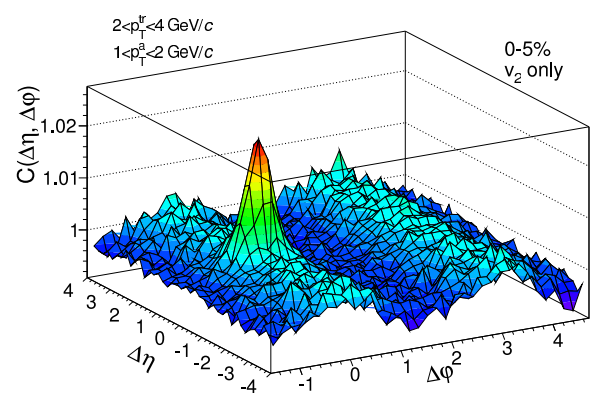

(a)

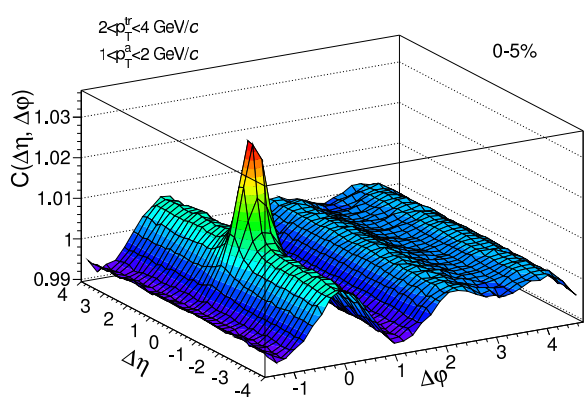

(b)

Figure 5. 2D correlation function in HYDJET ++ in $\mathrm{Pb}+\mathrm{Pb}$ collisions at $\sqrt{s_{\mathrm{NN}}}=2.76 \mathrm{TeV}$ for $2<p_{\mathrm{T}}^{\mathrm{tr}}<4 \mathrm{GeV} / \mathrm{c}$ and $1<p_{\mathrm{T}}^{\mathrm{a}}<2 \mathrm{GeV} / c$ for centrality $0-5 \%$ with (a) only elliptic flow and (b) with both elliptic and triangular flow.

elliptic flow from the quark coalescence at the partonic stage, most presumably, quark-gluon plasma (QGP). The violation of NCQ scaling was predicted by HYDJET++ $[25,26]$ before its experimental observation by the ALICE Collaboration [27].

The reduced functions $v_{2}\left(K E_{T} / n_{q}\right) / n_{q}$ of $\pi^{ \pm}, K^{ \pm}, p$ and $\Lambda$ calculated in HYDJET++ for 20-30\% central $\mathrm{Pb}+\mathrm{Pb}$ collisions at $2.76 \mathrm{TeV}$ are shown in Fig. 4. Left upper panel displays the results for the soft sector. As one can see, all distributions are indeed on the top of each other up to $1.5 \mathrm{GeV}$. To see the degree of the scaling fulfillment explicitly all particle flows in the bottom row are normalized to the flow of protons, $v_{2}^{h} / n_{q}: v_{2}^{p} / n_{q}$. Hadrons from jets make the scaling performance significantly worse, as seen im right panels of Fig. 4. The NCQ scaling at LHC holds only approximately within the range $0.4 \leq K E_{T} / n_{q} \leq 0.8 \mathrm{GeV}$ despite the fact that in pure hydrodynamic sector with final state interactions the scaling fulfillment is very good. The detailed study of consequences of the interplay between the soft processes and jets on the development of elliptic and triangular flow and higher order harmonics within the HYDJET++ can be found in [20, 25, 26, 28-31].

Finally, we would like to discuss the issue of correlations and fluctuations presented in the model. Among the sources of particle correlations in HYDJET++ are decays of resonances, jets, femtoscopic correlations, correlations imposed by energy-momentum conservation and so forth. However, as was shown in [32], the long-range correlations arise in the model merely because of the collective flow. The di-hadron correlation function $C(\Delta \eta, \Delta \varphi)$, where $\Delta \eta=\eta^{t r}-\eta^{a}$ and $\Delta \varphi=\varphi^{t r}-\varphi^{a}$ is presented in Fig. 5(a,b) for the calculations of $\mathrm{Pb}+\mathrm{Pb}$ collisions with centrality $0-5 \%$. - The indices " $\operatorname{tr}$ " and "a" indicate the so-called "trigger" and "associated" particle, respectively. - Two cases are considered: (a) without and (b) with the triangular flow in addition to the elliptic one. We see that the longrange azimuthal correlations appear both at near-side $(\Delta \varphi \approx 0)$ and at away-side $(\Delta \varphi \approx \pi)$ in both figures. But the experimentally observed $[33,34]$ double-hump structure at the away-side emerges in the model only in the presence of elliptic and triangular flow simultaneously.

The event-by-event (EbyE) distributions of anisotropic flow harmonics in $\mathrm{Pb}+\mathrm{Pb}$ collisions at $\mathrm{LHC}$ were studied by ATLAS Collaboration in [35] by means of the unfolding procedure. This method suppresses strongly the non-flow effects caused, e.g. by decays of resonances, finite event multiplicity or jet fragmentation. To get rid of such effects, unfolding was implemented in our study of the EbyE flow fluctuations within the HYDJET++ in [36]. Results are shown in Fig. 6, where the model calculations of probability density distributions of elliptic and triangular EbyE flow are compared with the ATLAS data. Agreement is good. Initial $P\left(V_{n}\right)$ distributions are broader than the unfolded ones; unfolding 


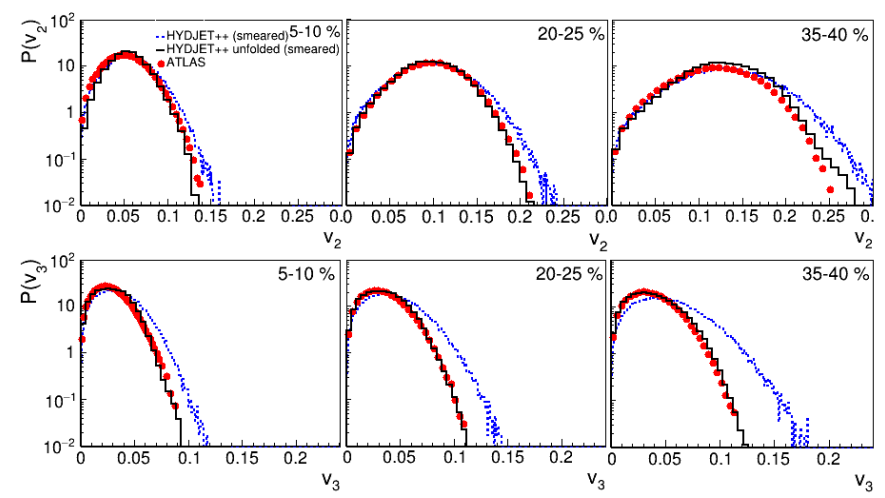

Figure 6. The probability density distributions of elliptic flow $V_{2}$ (upper row) and triangular flow $V_{3}$ (bottom row) in three centrality intervals: $5-10 \%$ (left), $20-25 \%$ (middle) and $35-40 \%$ (right). Dashed and solid histograms present the results for simulated HYDJET++ events before and after the unfolding procedure, respectively. The full circles are the ATLAS data from [35].

procedure makes it narrower. Obtained results strongly support the idea of dynamical origin of the EbyE flow fluctuations in the model.

\section{Conclusions}

We show the importance of hard processes for the correct treatment of anisotropic flow harmonics in heavy-ion collisions at ultrarelativistic energies within the ideal hydrodynamic model. Jets account for (i) falloff of the flow harmonics in the range of intermediate transverse momenta, (ii) changing of the mass ordering of the hadron elliptic and triangular flow, and (iii) the violation of the number-ofconstituent-quark scaling at energies of LHC and higher.

The interplay between $v_{2}$ and $v_{3}$ is able to describe both qualitatively and quantitatively the dihadron long-range correlations, i.e. ridge at near-side and double-hump structure at the away-side. The unfolding procedure was employed to study event-by-event flow fluctuations in the model and compare the obtained results with the experimental data. The comparison revealed dynamical origin of the flow fluctuations, originating primarily from the correlation between the momenta and coordinates of final particles and the velocities of hadronic fluid elements.

\section{Acknowledgments}

This work was supported in parts by the Grant INGO II LG15001 of the Ministry of Education, Youth and Sports of the Czech Republic and the Grant Agency of Czech Republic No.13-20841S, and the Grant of the President of Russian Federation for Scientific Schools Supporting No. 7989.2016.2. L.B. acknowledges financial support of the Alexander von Humboldt Foundation.

\section{References}

[1] E. Fermi, Prog. Theor.Phys. 5, 570 (1950) 
[2] G. Torrieri et al., Comput. Phys. Commun. 167, 229 (2005)

[3] A. Kisiel, T. Taluc, W. Broniowski, and W. Florkowski, Comput. Phys. Commun. 174, 669 (2006)

[4] L. D. Landau, Izv. Akad. Nauk Ser. Fiz. 17, 51 (1953);

S. Z. Belenkij and L. D. Landau, Nuovo Cim. Suppl. 3S10, 15 (1956)

[5] H. Song, S. A. Bass, and U. Heinz, Phys. Rev. C 83, 024912 (2011)

[6] B. Schenke, S. Jeon, and C. Gale, Phys. Rev. C 82, 014903 (2010)

[7] A. Capella, U. Sukhatme, C.-I. Tan, and J. Tran Thanh Van, Phys. Rep. 236, 225 (1994)

[8] S. A. Bass et al., Prog. Part. Nucl. Phys. 41, 255 (1998);

M. Bleicher et al., J. Phys. G 25, 1859 (1999)

[9] N. S. Amelin and L. V. Bravina, Sov. J. Nucl. Phys. 51, 133 (1990);

E. E. Zabrodin, C. Fuchs, L. V. Bravina, and Amand Faessler, Phys. Lett. B 508, 184 (2001);

J. Bleibel, L. V. Bravina, and E. E. Zabrodin, Phys. Rev. D 93, 114012 (2016)

[10] Z.-W. Lin, et al., Phys. Rev. C 72, 064901 (2005)

[11] I. P. Lokhtin et al., Comput. Phys. Commun. 180, 779 (2009)

[12] N. S. Amelin et al., Phys. Rev. C 74, 064901 (2006)

[13] N. S. Amelin et al., Phys. Rev. C 77, 014903 (2008)

[14] I. P. Lokhtin and A. M. Snigirev, Eur. Phys. J. C 46, 211 (2006)

[15] T. Sjostrand, S. Mrenna, and P. Skands, JHEP 0605, 026 (2006)

[16] R. Baier, Yu. L. Dokshitzer, A. H. Mueller, and D. Schiff, Phys. Rev. C 60, 064902 (1999)

[17] R. Baier, Yu. L. Dokshitzer, A. H. Mueller, and D. Schiff, Phys. Rev. C 64, 057902 (2001)

[18] S. A. Voloshin and Y. Zhang, Z. Phys. C 70, 665 (1996)

[19] S. Chatrchyan et al. (CMS Collaboration), Phys. Rev. C 87, 014902 (2013)

[20] L. V. Bravina et al., Eur. Phys. J. C 74, 2807 (2014)

[21] X.-N. Wang and M. Gyulassy, Phys. Rev. Lett. 68, 1480 (1992)

[22] J. Adam et al., (ALICE Collaboration), arXiv:1606.06057 [nucl-ex]

[23] J. Adams et al. (STAR Collaboration), Phys. Rev. Lett. 92, 052302 (2004)

[24] S. S. Adler et al. (PHENIX Collaboration), Phys. Rev. Lett. 91, 182301 (2003)

[25] G. Eyyubova et al., Phys. Rev. C 80, 064907 (2009)

[26] E. Zabrodin et al., J. Phys. G. 37, 094060 (2010)

[27] F. Noferini et al. (ALICE Collaboration), Nucl. Phys. A 904-905, 438c (2013)

[28] I. P. Lokhtin et al., Eur. Phys. J. C 72, 2045 (2012)

[29] L. Bravina, B. H. Brusheim Johansson, G. Eyyubova, and E. Zabrodin, Phys. Rev. C 87, 034901 (2013)

[30] L. V. Bravina et al., Phys. Rev. C 89, 024909 (2014)

[31] J. Crkovská et al., arxiv:1606.03250 [hep-ph]

[32] G. Eyyubova et al., Phys. Rev. C 91, 064907 (2015)

[33] K. Aamodt et al. (ALICE Collaboration), Phys. Rev. Lett. 107, 032301 (2011)

[34] S. Chatrchyan et al. (CMS Collaboration), Eur. Phys. J. C72, 2012 (2012)

[35] G. Aad et al. (ATLAS Collaboration), JHEP 11, 183 (2013)

[36] L. V. Bravina et al., Eur. Phys. J. C 75, 588 (2015) 